\title{
Plasma IP-10 could identify early lung disease in severe COVID-19 patients
}

\begin{abstract}
Dear Editor,
The global pandemic of SARS-Coronavirus-2 (SARSCoV-2) infection has imposed tremendous strain on healthcare resources worldwide, as a significant proportion of patients require intensive care. Although the majority have mild infections, up to $20 \%$ are estimated to become critically ill from severe disease. ${ }^{1}$ Age, concurrent comorbidities, more severe disease, respiratory failure, higher levels of D-dimer and C-reactive protein (CRP), more severe lymphopaenia, and secondary infections are associated with risk of mortality. ${ }^{2}$
\end{abstract}

Innate and adaptive immune responses to COVID-19 contribute to disease pathology. Elevated levels of circulating interleukin 1 beta, interferon gamma, CXCL10/interferon gamma inducible protein-10 (IP-10) and CCL2/monocyte chemoattractant protein 1 have been associated with mild disease. Deterioration of disease leading to intensive care and adverse outcome of COVID-19 has been associated with persistently high levels of chemokines such as IP-10. ${ }^{3}$ Thus we explored the potential utilisation of plasma IP-10 in conjunction with clinical, imaging and laboratory parameters in assisting risk stratification for disease progression of COVID-19, because of the role this chemokine has in regulating the interferon response and innate immunity.

A total of 72 de-identified patients at the National Centre of Infectious Diseases, Singapore with confirmed COVID-19 by nasopharyngeal swab SARS-CoV-2 real-time reverse transcriptase-polymerase chain reaction (RT-PCR), were recruited over the period 28 March to 1 April 2020. Cytokine assays were performed on frozen sera from blood samples collected for standard clinical evaluation, with waiver of consent approved by institutional review board (DSRB 2020/00910). Data on demographics, comorbidities, and laboratory results were obtained from electronic medical records. The Charlson comorbidity index was calculated. ${ }^{4}$ Plasma IP-10 levels were determined by ELISA after viral inactivation by incubation to a final concentration of $1 \%$ Triton X-100. Levels from 50 healthy controls were used for comparison.

Correlation of clinical, laboratory, imaging and cytokine data was performed with non-parametric Fisher exact test and Mann-Whitney U test for univariate comparisons. Correlation of levels of plasma IP-10 with levels of CRP, lactate dehydrogenase (LDH), platelets, lymphocytes, neutrophils and RT-PCR cycle threshold values was assessed using Spearman correlation. Diagnostic usefulness in predicting intensive care unit (ICU) admission was evaluated by formulating a logistic model using variables showing statistical significance in univariate analysis. The receiver operator characteristic (ROC) curve of the model was then generated to determine the predictive performance of the model as reflected by the area under the ROC curve.

The patients' clinical characteristics, disease severity, relevant laboratory data, imaging and course, including stay in intensive care, are shown in Table 1. Disease severity varied. Most had fever $(37 / 72,51 \%)$ and cough $(31 / 72,43 \%)$; other symptoms included myalgia $(6 / 72,8 \%)$, sore throat $(5 / 72,7 \%)$, anosmia $(4 / 72$, $6 \%$ ), rhinorrhoea $(4 / 72,6 \%)$, diarrhoea $(2 / 72,3 \%)$ and headache $(1 / 72,1 \%)$. Thirty patients $(41.6 \%)$ exhibited abnormal chest X-ray changes ranging from opacities to consolidation.

Plasma samples that were assayed for IP-10 levels were drawn from 1 to 22 (mean 7.8 \pm 4.5 ) days after symptom onset. Significantly higher median plasma levels of IP-10 were found in SARS-CoV-2 patients than in healthy controls: 95.8 (interquartile range [IQR] 45.7-195.1) $\mathrm{pg} / \mathrm{mL}$ versus 22.1 (IQR 9.4-38.1) $\mathrm{pg} / \mathrm{mL}$ $(P<0.001)$. The correlations between IP-10 levels and the laboratory parameters are summarised in Table 1 . We observed that plasma IP-10 levels correlated positively with CRP $(\mathrm{r}=0.809, P<0.005), \mathrm{LDH}$ $(\mathrm{r}=0.341, P<0.005)$, neutrophil count $(\mathrm{r}=0.520$, $P<0.001)$ and negatively with lymphocyte count $(\mathrm{r}=-0.292, P=0.013)$. SARS-CoV-2 patients with chest X-ray changes exhibited higher plasma IP-10 levels than those without. In addition, a positive correlation was observed between IP-10 levels and multiple comorbidities based on the Charlson Comorbidity Index ( $\mathrm{r}=0.491, P<0.001$, Table 1$)$. IP10 levels did not correlate with viral load based on SARS-CoV-2 RT-PCR cycle threshold values, likely as a result of the RT-PCR assays being done later in the disease course, while the CRP, LDH and blood counts were done contemporaneously with the IP-10 assays.

We next evaluated predictors of ICU admission. ICU admission was found to be significantly correlated in univariate analysis with the Charlson Comorbidity Index $(P<0.01)$, thrombocytosis (platelet $>300 \times 10^{9} / \mathrm{L}$, 
Table 1. Patient demographics and clinical characteristics, IP-10 plasma levels and clinical correlations

\begin{tabular}{|c|c|c|}
\hline Demographics and clinical characteristics & \multicolumn{2}{|c|}{$\begin{array}{c}\text { SARS-CoV-2 patients }(\mathrm{N}=72) \\
\text { Mean } \pm \text { SD (IQR) }\end{array}$} \\
\hline Age, years & \multicolumn{2}{|c|}{$45.5 \pm 16.5(30.8-58.0)$} \\
\hline Male/Female, no. (\%) & \multicolumn{2}{|c|}{$41(57) / 31(43)$} \\
\hline Day of illness & \multicolumn{2}{|c|}{$7.8 \pm 4.5(4-10)$} \\
\hline Abnormal CXR (opacities/consolidation), no. (\%) & \multicolumn{2}{|c|}{$30 / 72(41.6)$} \\
\hline ICU admission, no. (\%) & \multicolumn{2}{|c|}{$7 / 72(9.7)$} \\
\hline CRP mg/L & \multicolumn{2}{|c|}{$27.0 \pm 50.4(3.0-23.6)$} \\
\hline LDH U/L & \multicolumn{2}{|c|}{$479.0 \pm 355.2(359.8-487.3)$} \\
\hline Lymphocyte count, $10^{9} / \mathrm{L}$ & \multicolumn{2}{|c|}{$1.3 \pm 0.5(0.9-1.6)$} \\
\hline Neutrophil count, $10^{9} / \mathrm{L}$ & \multicolumn{2}{|c|}{$3.5 \pm 1.9(2.2-4.2)$} \\
\hline Platelet count, $10^{9} / \mathrm{L}$ & \multicolumn{2}{|c|}{$219.2 \pm 79.9(165.5-258.8)$} \\
\hline SARS-CoV-2 RT-PCR cycle & \multicolumn{2}{|c|}{$29.6 \pm 6.1(25.1-34.3)$} \\
\hline IP-10pg/mL & \multicolumn{2}{|c|}{$163.1 \pm 195.6(45.7-197.0)$} \\
\hline IP-10 plasma levels and clinical correlations & Pearson correlation coefficient & $P$ value \\
\hline IP-10 and CRP & 0.809 & $0.005^{\mathrm{a}}$ \\
\hline IP-10 and LDH & 0.341 & $0.005^{\mathrm{a}}$ \\
\hline IP-10 and lymphocyte count & -0.292 & $0.013^{\mathrm{a}}$ \\
\hline IP-10 and neutrophil count & 0.520 & 0.001 \\
\hline IP-10 and Charlson score & 0.491 & $0.001^{\mathrm{a}}$ \\
\hline IP-10 and COVID-19 RT-PCR cycle & -0.059 & 0.656 \\
\hline
\end{tabular}

${ }^{a}$ Correlation is significant at the 0.05 level (2-tailed)

CRP: C-reactive protein; CXR: chest X-ray; ICU: intensive care unit; IP-10: interferon gamma inducible protein-10; IQR: interquartile range;

LDH: lactate dehydrogenase; RT-PCR: reverse transcriptase-polymerase chain reaction; SD: standard deviation

$P<0.01)$, high CRP $(>50 \mathrm{mg} / \mathrm{L}, P<0.05)$, and high IP-10 level $(\geq 200 \mathrm{pg} / \mathrm{mL}, P<0.05)$. A logistic regression model using the laboratory data with significant univariate association with ICU admission (CRP, thrombocytosis and high IP-10 level) was found to have an area under the ROC curve of 0.9546 , implying a strong predictive performance.

SARS-COV-2 is a zoonotic RNA betacoronavirus. Infection with SARS-CoV can trigger an exaggerated immune response with excessive production of chemokines including IP-10, leading to inflammation and destruction of lung tissue through the infiltration of neutrophils, alveolar macrophages and Th1 lymphocytes. ${ }^{5}$ This may also be the case with SARS-Cov-2 as elevated IP-10 levels have been reported to be associated with prolonged fever, increased hypoxia and disease severity in COVID-19 patients. ${ }^{6-8} \mathrm{~A}$ recent observational cohort study of 52 hospitalised patients from Israel found that IP-10 levels were higher in patients with severe disease and those requiring ICU admission, findings in keeping with those of our study. ${ }^{9}$

The limitation of our cross-sectional study is that levels of IP-10 were performed an average of 7 days after symptom onset, thus the utility of IP-10 for early risk stratification is not fully known.

The addition of blood IP-10 levels to the risk factor profile may hold promise for early identification of individuals with oncoming severe COVID-19 lung disease, facilitating the institution of therapy that could potentially avert intensive care and prevent mortality, Larger, adequately powered longitudinal studies will be required for this to be determined. 


\section{REFERENCES}

1. Puah SH, Young BE, Chia PY, et al. Clinical features and predictors of severity in COVID-19 patients with critical illness in Singapore. Sci Rep 2021;11:7477.

2. Young BE, Ong SWX, Kalimuddin S, et al. Epidemiologic Features and Clinical Course of Patients Infected With SARS-CoV-2 in Singapore. JAMA 2020;323:1488-94.

3. Lei R, Mohan C. Immunological biomarkers of COVID-19. Crit Rev Immunol 2020;40:497-512.

4. Charlson ME, Pompei P, Ales KL, et al. A new method of classifying prognostic comorbidity in longitudinal studies: development and validation. J Chronic Dis 1987;40:373-83.

5. Chu H, Chan JF, Wang Y, et al. Comparative replication and immune activation profiles of SARS-CoV-2 and SARS-CoV in human lungs: an ex vivo study with implications for the pathogenesis of COVID-19. Clin Infect Dis 2020;71:1400-9.

6. Lam CW, Chan MH, Wong CK. Severe acute respiratory syndrome: clinical and laboratory manifestations. Clin Biochem Rev 2004;25:121-32.

7. Yang Y, Shen C, Li J, et al. Plasma IP-10 and MCP-3 levels are highly associated with disease severity and predict the progression of COVID-19. J Allergy Clin Immunol 2020;146:119-27.e4.

8. Chen Y, Wang J, Liu C, et al. IP-10 and MCP-1 as biomarkers associated with disease severity of COVID-19. Mol Med 2020;26:97
9. Lev S, Gottesman T, Sahaf Levin G, et al. Observational cohort study of IP-10's potential as a biomarker to aid in inflammation regulation within a clinical decision support protocol for patients with severe COVID-19. PLoS One 2021;16:e0245296.

Hwee Siew Howe ${ }^{1}{ }_{M M e d}$, Li Min Ling ${ }^{2}{ }^{2}{ }{ }^{2}$, Elampirai Elangovan ${ }^{2}{ }_{M B B S}$, Shawn $\underline{\text { Vasoo }}^{2}{ }^{2}{ }^{2} P$, Mohammad Yazid $\underline{\mathrm{Abdad}}^{2}{ }^{2} \mathrm{~h}$, Bernard Yu Hor Thong ${ }^{1}$ FRCP, Kok Ooi Kong ${ }^{1}{ }_{M R C P}$, Bernard Pui Lam Leung ${ }^{1,3}$ PhD

${ }^{1}$ Department of Rheumatology, Allergy and Immunology, Tan Tock Seng Hospital, Singapore

${ }^{2}$ National Centre for Infectious Diseases, Singapore

${ }^{3}$ Singapore Institute of Technology, Singapore

Correspondence: Dr Hwee Siew Howe, Department of Rheumatology, Allergy and Immunology, Tan Tock Seng Hospital, 11 Jalan Tan Tock Seng, Singapore 308433.

Email: hwee_siew_howe@ttsh.com.sg 